\title{
Comparison of in vivo antidiabetes activity of snake fruit Kombucha, black tea Kombucha and metformin
}

\author{
Elok Zubaidah $^{\mathrm{a}, *}$, Chairul Anam Afgani ${ }^{\mathrm{b}}$, Umi Kalsum ${ }^{\mathrm{b}}$, Ignatius Srianta ${ }^{\mathrm{c}}$, Philippe J. Blanc ${ }^{\mathrm{d}}$ \\ ${ }^{a}$ Department of Food Science and Technology, Faculty of Agricultural Technology, Brawijaya University, Jalan Veteran Malang, 65145, Indonesia \\ ${ }^{\mathrm{b}}$ Department of Pharmacology, Faculty of Medicine, Brawijaya University, Jalan Veteran Malang, 65145, Indonesia \\ ${ }^{\mathrm{c}}$ Department of Food Technology, Faculty of Agricultural Technology, Widya Mandala Catholic University Surabaya, Jalan Dinoyo 42-44, Surabaya 60265, Indonesia \\ ${ }^{\mathrm{d}}$ Université de Toulouse, INSA, LISBP, CNRS, UMR5504, INRA, UMR792, Ingénierie des Systèmes Biologiques et des Procédés, 135 Avenue de Rangueil, F-31077 \\ Toulouse, France
}

A R T I C L E I N F O

\section{Keywords:}

Diabetes Mellitus

Fermentation

Kombucha

Pancreatic $\beta$-cells

Fasting plasma glucose

\begin{abstract}
A B S T R A C T
The research compared antidiabetic activity of snake fruit Kombucha, black tea Kombucha and metformin in streptozotocin-induced diabetic rats. Snake fruit Kombucha, black tea Kombucha and metformin were orally administered to the diabetic rats daily during a 28-day experiment. Fasting plasma glucose (FPG) levels, superoxide dismutase (SOD) activities, malondialdehyde (MDA) levels and lipid profiles (total triglyceride, total cholesterol, LDL-cholesterol and HDL-cholesterol) of the blood plasma were investigated. Pancreas immunohistochemical study and $\beta$-cells quantification were also conducted. The products significantly $(\mathrm{p}<0.05)$ reduced fasting plasma glucose levels (67-76\%) and improved oxidative stress indices and lipid profiles. From immunohistochemical staining of pancreatic tissues, pancreatic $\beta$-cells were also improved in the diabetic rats by the products. The snake fruit Kombucha compared with the metformin but better than the black tea Kombucha as a diabetes therapy agent.
\end{abstract}

\section{Introduction}

Diabetes mellitus (DM) is a metabolic disorder characterized by hyperglycemia with polyphagia, polyuria, more eating and body weight drop, and it is known as a silent killer. It is one of the biggest health problem globally (Ogurtsova et al., 2017). Without proper therapeutic management, it leads to severe complications and death. However, it is widely managed by insulin injection and drugs, but natural functional foods without negative effects are being investigated as substitutes, and Kombucha is becoming an appealing alternative (Zubaidah et al., 2018a).

Kombucha is processed by fermentation with symbiotic associations of bacteria and yeast (Kombucha consortium), it is generally made from black tea and has bioactive compounds with antidiabetic activity by inhibiting $\alpha$-amylase activity and suppressing blood glucose levels (Dufresne and Farnworth, 2000; Goh et al., 2012; Bhattacharya et al., 2013; Srihari et al., 2013; Jayabalan et al., 2014). Those positive health properties have led to research on other substrates (e.g. snake fruit) for its manufacture. Snake fruit is a tropical fruit that contains vitamins, minerals, dietary fiber, and bioactive compounds with antioxidant activities (Aralas et al., 2009; Suica-Bunghez et al., 2016). Previous studies from our laboratory have demonstrated antioxidant and antidiabetic properties of Kombucha from snake fruits (Zubaidah et al., 2018a, 2018b) to stir further research interests on its comparative antidiabetic advantages. However, most of these studies were done without comparing the efficacy of the functional foods with diabetes drugs, such as metformin. Such is necessary to understand the relative effectiveness of the intervention. Therefore, the objective of this study was to compare snake fruit and black tea Kombucha, and metformin as diabetes therapy agents.

\section{Materials and methods}

\subsection{Materials}

Snake fruit (Salak Suwaru cultivar) of commercial maturity, commercial Kombucha starter, black tea, and cane sugar were obtained from plantations, distributors and supermarkets in Malang, East Java, Indonesia as described before (Zubaidah et al., 2018a, 2018b). The Salak Suwaru cultivar of the snake fruit was chosen because it showed the best characteristics out of the 4 cutivars (Salak Suwaru, Salak Doyong, Salak Pondoh and Salak Segaran) we had studied to date

\footnotetext{
* Corresponding author.

E-mail addresses: elok@ub.ac.id, elzoeba@yahoo.com (E. Zubaidah).
} 
(Zubaidah et al., 2018a, 2018b). Metformin was obtained from Pharmacology Laboratory, Faculty of Medicine, Brawijaya University.

\subsection{Kombucha preparation and analysis}

The snake fruit was juiced and its Kombucha was prepared following the procedure as previously described (Zubaidah et al., 2018a). Black tea Kombucha was prepared according to Ardheniati et al. (2009) by extracting $10 \mathrm{~g}$ of the black tea in $500 \mathrm{~mL}$ of boiling water for $10 \mathrm{~min}$ prior to filtering and sweetening (10\% sugar). The sugared tea extract was put into a glass jar, cooled to room temperature before adding $10 \%$ Kombucha starter aseptically. The jar was then covered with a sterile cheese cloth and incubated at room temperature for 14 days. The physicochemical and antioxidant properties of the Kombucha were analyzed as before (Zubaidah et al., 2018a).

\subsection{Animal experiment and analysis}

Twenty five healthy three-months old male Wistar rats were divided randomly into 5 groups with essentially 5 replicates - group 1 (P0), normal; group 2, diabetic, DM (P1); group 3, DM with the black tea Kombucha (KT) at a dose of $5 \mathrm{~mL} / \mathrm{kg} \mathrm{BW} /$ day (P2); group 4, DM with the snake fruit Kombucha (KS) at a dose of $5 \mathrm{~mL} / \mathrm{kg} \mathrm{BW} /$ day (P3); and group 5, DM with the metformin at the recommended dose of $45 \mathrm{mg} / \mathrm{kg}$ $\mathrm{BW} /$ day (P4). The DM rats were induced with streptozotocin (Nacalai Tesque, Japan) intraperitoneally at a dose of $45 \mathrm{mg} / \mathrm{kg} \mathrm{BW}$. The rats accessed standard diet and water ad libitum during the 28-day experiment, and groups 3-5 were respectively administered with tea Kombucha, snake fruit Kombucha and metformin on a regular basis once a day.

Fasting plasma glucose (FPG) level measurements were conducted on days 0 and 28, and at the end of the experiment, the rats were sacrificed by cervical dislocation. The blood was used to analyse for superoxide dismutase (SOD) activity, malondialdehyde (MDA) and lipid profiles (total cholesterol, triglycerides, high-density lipoprotein (HDL) cholesterol, and low-density lipoprotein (LDL) cholesterol levels) as before (Zubaidah et al., 2018b).

\subsection{Immunohistochemical staining (IHC)}

After sacrifice, the rat pancreas was taken and fixed in $10 \%$ buffered formalin for $24 \mathrm{~h}$, and slides were made by standard methods using paraffin. IHC staining was done as in Beesley (1995), and it involved treating with diamino benzidine (DAB) for 3 min and counter staining with mayers haematoxilin for $3 \mathrm{~min}$. Insulin was visualized as brown color. Quantification of $\beta$-cells was conducted according to Suarsana et al. (2010) by calculating the average of $\beta$-cells.

\subsection{Statistical analysis}

The data were analyzed by analysis of variance (ANOVA), followed by the LSD test at $\mathrm{p}<0.05$.

\section{Results and discussion}

Kombucha fermentation is a biochemical process, whereby the Kombucha consortium transforms substrates into products such as acetic acid and others. Table 1 shows the characteristics of the Kombucha, and the differences before and after the fermentation are expected from the microbial activities of the Kombucha consortium on sucrose, which is the main carbon source (Malbasa et al., 2011; Jasman and Widianto, 2012). Both Kombucha decreased in $\mathrm{pH}$, total sugar and total solids and increased in total acidity, total phenolic content, tannin content, and total antioxidant activity; consistent with previous studies (Kallel et al., 2012; Johnson and De Mejia, 2016). The total acidity in the snake fruit Kombucha was higher than that in the black tea, possibly due to the higher natural organic acids in snake fruits, while the differences in total phenolic content, tannin content and antioxidant activity might be related to the differences in the raw materials; the sugared black tea extract and the sugared snake fruit juice.

\subsection{Fasting plasma glucose (FPG) level}

Changes in the FPG levels before and after the treatment are presented in Fig. 1. The FPG levels among the DM rat groups (P1, P2, P3, and P4) on day 0 are not significantly different ( $p>0.05$ ), while there are significant differences $(\mathrm{p}<0.05)$ with the normal rats $(\mathrm{P} 0)$. At the end of the 28-day treatment, the normal and DM rats appeared to show constant FPG levels, whereas the FPG levels of the DM groups P2, P3 and P4 decreased. The DM group with the KS (P3) and metformin (P4) administration showed lower FPG levels than the DM group with the KT (P2).

Metformin is an antidiabetic drug, and it works by increasing the sensitivity of the liver and peripheral tissues to insulin without affecting insulin secretion and increasing glucose uptake in the peripheral tissues to reduce insulin resistance (Iida et al., 2003). The snake fruit Kombucha decreased the level of FPG, because of its high content of antioxidant compounds such as phenolic, tannin and other bioactives, as well as some other organic acids such as citric, lactic, butyric, and propionic acids (Ostman et al., 2005; Zubaidah et al., 2018a). Antioxidant compounds decrease FPG levels through increasing cellular glucose uptake. Increased insulin secretion will have implications for the body's ability to utilize blood glucose for normal metabolism. Glycolysis, glycogenesis and lipogenesis are some of the metabolisms of insulin-regulated glucose (Dufresne and Farnworth, 2000; Aloulou et al., 2012). Antioxidant compounds can also inhibit glucose absorption in the small intestine by decreasing glucose-conducting activity, such as sodium-glucose transport protein 1 (GLUT1), glucose transporter 5 (GLUT 5) and glucose transporter 2 (GLUT 2). The gross effect of these is to reduce the glucose that enters the bloodstream (Kwon et al., 2007). Consequently, it can be inferred that the snake fruit Kombucha had a comparable effect as the metformin and a better effect on FPG than the black tea Kombucha.

\subsection{Superoxide dismutase (SOD) activity and malondialdehyde (MDA) levels}

The SOD activity and MDA levels reflect the body oxidation status (Bhattacharya et al., 2013), and Table 2 shows that the rats in the DM group had higher oxidative stresses than the rats in the normal group, with the Kombucha and metformin administered rats having higher SOD activities and lower MDA levels than the DM group to indicate improved oxidation status in the DM rats. This is expected as the antioxidant compounds, polyphenols (Table 1) in the Kombucha would have contributed to this desirable outcome. The potent antioxidant ability of phenolic compounds is thought to be due to the ability to donate electrons or hydrogen atoms from the -OH group, which makes them unstable but the resonance of electrons by the benzene ring stabilizes them for their desirable properties (Scalbert et al., 2005). This enhances Kombucha contributions to the body antioxidant enzyme system thereby reducing the adverse effects of diabetes mellitus (Zhang and Tsao, 2016). It is noteworthy that the Kombuchas were either comparable or better than the metformin in these body oxidation indices (Table 2).

\subsection{Lipid profiles}

Table 3 shows the total cholesterol, total triglyceride and LDL-c of the diabetic rat groups were higher than those of the normal rat group, due to lipid metabolism disorders. Administering the Kombuchas and metformin to the diabetic rat groups improved the lipid profiles, and the snake fruit Kombucha was more effective in the improvement than 
Table 1

Chemical characteristics of the black tea Kombucha and snake fruit Kombucha\# ${ }^{\#}$

\begin{tabular}{|c|c|c|c|c|}
\hline \multirow[t]{3}{*}{ Parameter } & \multicolumn{4}{|l|}{ Kombucha Type } \\
\hline & \multicolumn{2}{|c|}{ Black tea Kombucha } & \multicolumn{2}{|c|}{ Snake fruit Kombucha } \\
\hline & Day 0 & Day 14 & Day 0 & Day 14 \\
\hline Total acidity (\%) & $0.09 \pm 0.04$ & $0.42 \pm 0.07 * *^{\mathrm{a}}$ & $0.57 \pm 0.14$ & $1.56 \pm 0.17 * * \mathrm{~b}$ \\
\hline $\mathrm{pH}$ & $4.77 \pm 0.26$ & $3.08 \pm 0.10 * * \mathrm{a}$ & $3.91 \pm 0.19$ & $3.22 \pm 0.09 * * \mathrm{a}$ \\
\hline Total sugar $(\%)$ & $9.06 \pm 1.01$ & $6.78 \pm 0.06 * * \mathrm{a}$ & $10.50 \pm 0.44$ & $7.76 \pm 0.03 * * \mathrm{a}$ \\
\hline Total solid (\%) & $9.90 \pm 0.10$ & $8.13 \pm 0.06 * * \mathrm{a}$ & $13.93 \pm 0.06$ & $12.88 \pm 0.08 * * \mathrm{~b}$ \\
\hline Total phenolic content (mg/L GAE) & $228.15 \pm 24.16$ & $400.06 \pm 47.99 * * a$ & $281.01 \pm 11.28$ & $535.59 \pm 1.96 * * b$ \\
\hline Tannin (mg/L TAE) & $530.89 \pm 82.51$ & $704.81 \pm 32.25 * *^{\mathrm{a}}$ & $496.67 \pm 7.64$ & $619.00 \pm 39.15 * * b$ \\
\hline Antioxidant activity/DPPH (\%) & $79.82 \pm 4.94$ & $89.33 \pm 1.25 * * \mathrm{a}$ & $86.38 \pm 1.18$ & $91.73 \pm 3.64 * * \mathrm{~b}$ \\
\hline
\end{tabular}

\#Values are means \pm standard deviations $(\mathrm{n}=3$ ). GAE = Gallic acid equivalent, TAE = Tannic acid equivalent, DPPH = 2,2-diphenyl-1-picrylhydrazyl.

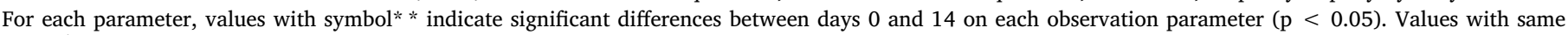
letter $^{\mathrm{a}, \mathrm{b}}$ indicate no significance differences between snake fruit Kombucha and black tea Kombucha on each parameter ( $\mathrm{p}>0.05$ ).

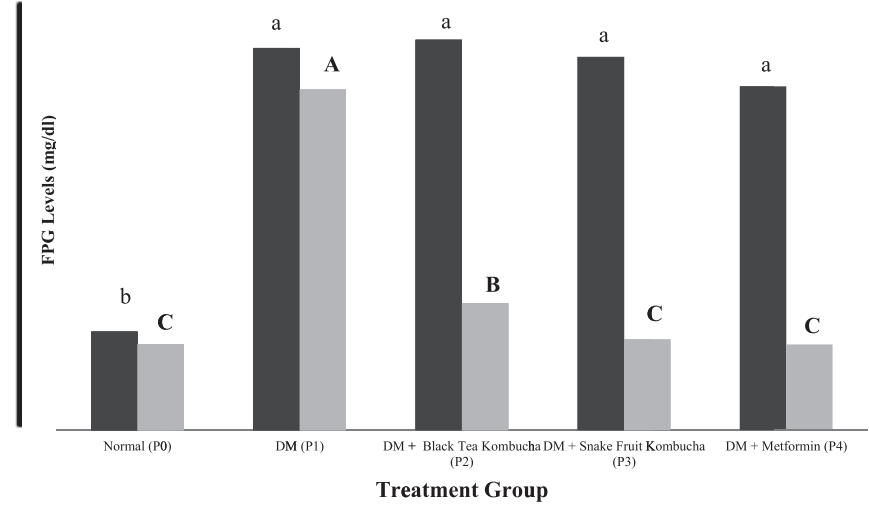

Fig. 1. Effect of the Kombucha and metformin administration on indices of the fasting plasma glucose (FPG) levels in the rats. Values are means \pm SD $(n=4)$. Bars with the same letters are not significantly different $(\mathrm{p}>0.05)$. Lower case letters are for day 0 , while capital letters are for day 28.

Table 2

Effect of the black tea Kombucha, snake fruit Kombucha and metformin administration on SOD activity and MDA level in rats.

\begin{tabular}{lll}
\hline Treatment & SOD (unit/100 $\boldsymbol{\mu L})$ & MDA (ng/100 $\boldsymbol{\mu L})$ \\
\hline P0 (Normal) & $52.47 \pm 2.23^{\mathrm{a}}$ & $0.28 \pm 0.03^{\mathrm{d}}$ \\
P1 (DM) & $17.66 \pm 4.79^{\mathrm{d}}$ & $0.83 \pm 0.02^{\mathrm{a}}$ \\
P2 (DM + KT) & $39.50 \pm 11.71^{\mathrm{b}}$ & $0.44 \pm 0.02^{\mathrm{bc}}$ \\
P3 (DM + KS) & $44.55 \pm 5.98^{\mathrm{b}}$ & $0.46 \pm 0.02^{\mathrm{bc}}$ \\
P4(DM + Metformin) & $31.78 \pm 3.79^{\mathrm{c}}$ & $0.39 \pm 0.02^{\mathrm{c}}$ \\
\hline
\end{tabular}

DM: Diabetes Mellitus, KT: Black tea Kombucha KS: Snake fruit Kombucha.

Values in a column with the same letters are not significantly $(\mathrm{p}>0.05)$ different.

This applies to all tables, where they appear.

the black tea Kombucha, possibly due to its higher bioactive compounds (Table 1) as discussed above. The snake fruit Kombucha also significantly showed better effects than the metformin (Table 3).

The mechanism of phenolic compounds in improving lipid profiles is through donating hydrogen atoms to lipid radicals ( $\left.\mathrm{R}^{*}, \mathrm{ROO} *\right)$ and converting them to a more stable form and slowing the auto-oxidation rate. The addition of a low concentration of primary antioxidants to lipids reportedly inhibits or prevents oxidation of fatty acids (Gordon, 1990; Taku et al., 2007). Phenolic compounds can also increase HDL-c and lower total cholesterol, triglycerides, and LDL-c by aiding metabolisms in generating energy, contributing to fat metabolisms, increasing bile acid excretions, decreasing total cholesterol absorptions by binding cholesterol carriers as they pass through the brush border membranes. This consequently decreases the production of lipoproteins and increases the activity of lecithin cholesterol acyl transferase (LCAT). LCAT is an enzyme that converts free cholesterol to cholesterol esters and plays a role in HDL-c metabolisms (Carvajall-Zarrabal et al., 2005; Zern and Fernandez, 2005; Tan et al., 2007; Rahimi-Madiseh et al., 2017).

\subsection{Pancreas immunohistochemistry (IHC) study}

The result of IHC staining is shown in Figs. 2 and 3. The results showed increasing Langerhans island structures and insulin secretions in the three treated (Kombucha and metformin) groups (Fig. 3). The size and shape of the structures from the DM group were irregular and smaller than those of the normal group (P0) and the three groups of KT, KS and metformin. In addition, the DM group showed a very low immunoreactive response (brown color) to anti-insulin, which indicated low levels of insulin production. The DM rat groups with the Kombucha and metformin improved in the Langerhans island structures, and in the size, shape, distributions, and numbers of the $\beta$-cells, as well as the high intensity of the brown color when compared with the DM rats (Fig. 3).

The DM group with metformin (P4) had a high number of the pancreatic $\beta$-cells, which is not significantly $(p>0.05)$ different from the snake fruit Kombucha. Metformin has adenosine monophosphateactivated protein kinase (AMPK) enzyme that plays a role in repairing HbA1c, the main parameter of blood glucose. In addition, it can reduce hepatic glucose production, lower LDL and triglyceride levels, increase HDL levels, decrease platelet aggregation, increase fibrinolytic activity and improve weight, reduce the risk of hypoglycemia, and increase insulin sensitivity with a concomitant improvement of pancreatic

Table 3

Effect of the black tea Kombucha, snake fruit Kombucha and metformin administration on the lipid profile serum levels inthe rats.

\begin{tabular}{|c|c|c|c|c|}
\hline Treatment & Cholesterol (mg/dL) & Triglycerides (mg/dL) & HDL (mg/dL) & LDL (mg/dL) \\
\hline P0 (Normal) & $44.75 \pm 11.22 \mathrm{~b}$ & $48.00 \pm 21.12 c$ & $66.75 \pm 1.71 \mathrm{a}$ & $6.75 \pm 6.29 c$ \\
\hline P1 (DM) & $75.25 \pm 10.50 \mathrm{a}$ & $102.75 \pm 22.94 \mathrm{a}$ & $37.75 \pm 5.70 \mathrm{c}$ & $14.25 \pm 3.40 \mathrm{a}$ \\
\hline $\mathrm{P} 2(\mathrm{DM}+\mathrm{KT})$ & $51.50 \pm 5.20 c$ & $73.25 \pm 24.53 b$ & $40.50 \pm 9.95 b$ & $11.50 \pm 1.91 \mathrm{~b}$ \\
\hline P3 (DM+KS) & $44.75 \pm 3.79 b$ & $52.50 \pm 28,84 c$ & $58.75 \pm 2.87 \mathrm{a}$ & $7.25 \pm 1.26 \mathrm{c}$ \\
\hline P4 (DM + Metformin) & $52.50 \pm 9.00 c$ & $91.75 \pm 26.71 b$ & $56.75 \pm 21.65 \mathrm{a}$ & $11.00 \pm 0.82 \mathrm{~b}$ \\
\hline
\end{tabular}



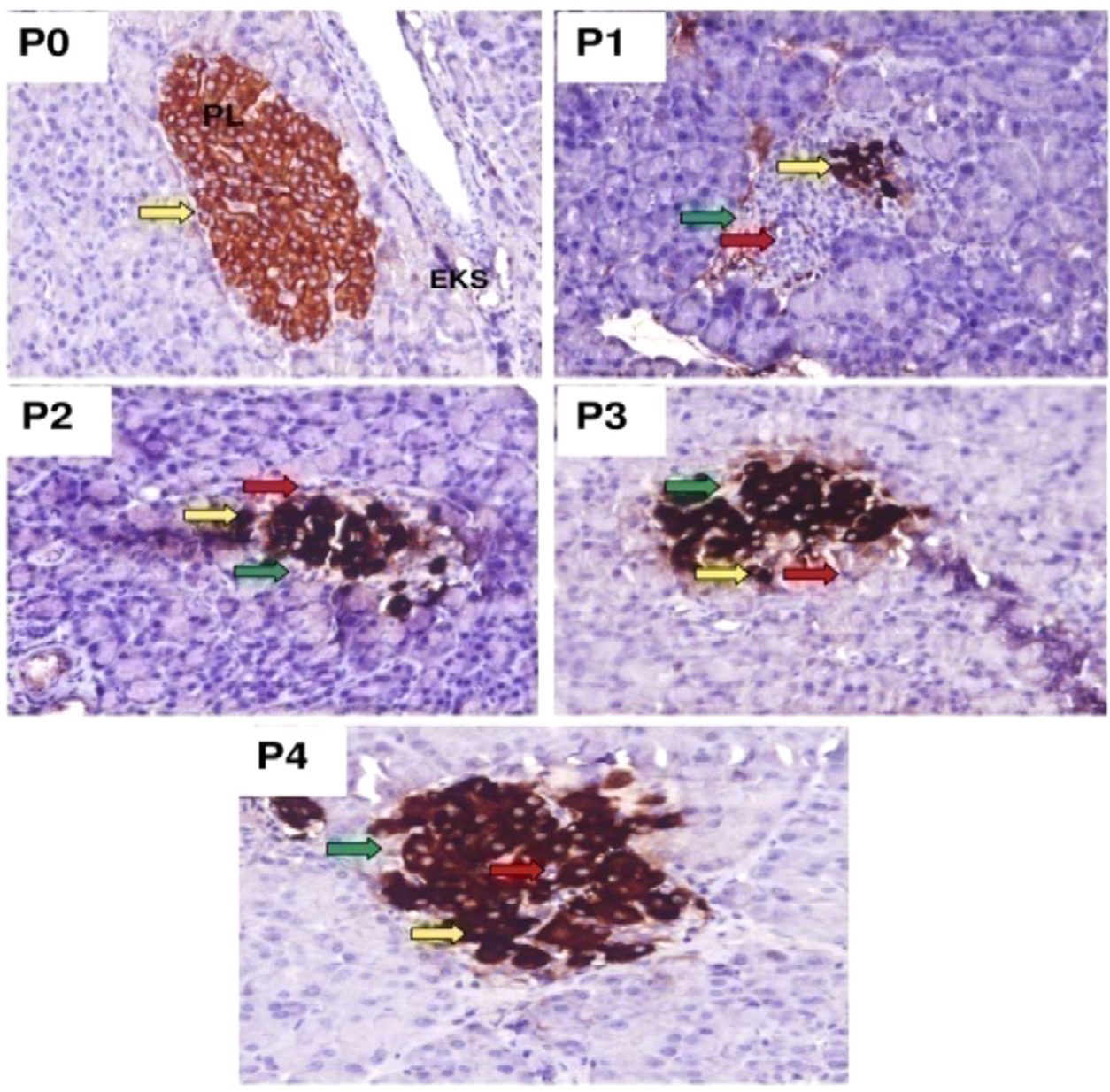

Fig. 2. Effect of the Kombucha and metformin administration on the pancreatic cells in rats evaluated by the IHC staining (400 $\times$ magnification). PL: Langerhans Island, EKS: Exocrine glands (acini). Yellow arrow: Pancreatic $\beta$-cells, which have immunoreactivity to anti-insulin. Green arrow: Endocrine cells, which are not immunoreactive to anti-insulin. Red arrow: Empty space by necrosis, DM: Diabetes Mellitus, KT: black tea Kombucha, KS: snake fruit Kombucha, BW: Body Weight. (For interpretation of the references to color in this figure legend, the reader is referred to the web version of this article).

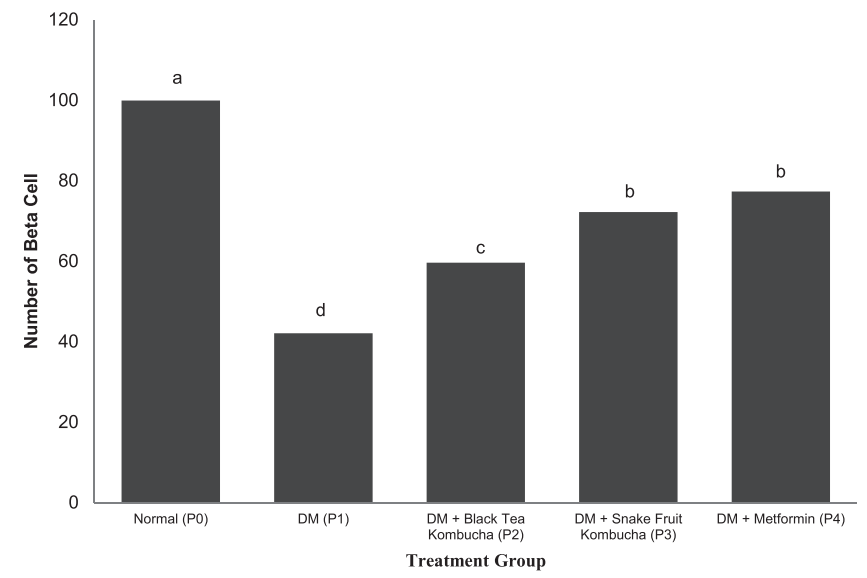

Fig. 3. Effect of the Kombucha and metformin administration on the number of pancreatic $\beta$-cells. Values are mean \pm SD of $\beta=$ cells of five Langerhans islands. The same letter indicates no significant difference $(\mathrm{p}>0.05)$. DM: Diabetes Mellitus.

performances (Gunton et al., 2003). The DM group with the snake fruit Kombucha treatment had the pancreatic $\beta$-cells higher than those of the black tea Kombucha, possibly due to its higher antioxidant compounds as discussed above that can protect and repair the pancreatic $\beta$-cells and increase insulin secretions (Zubaidah et al., 2018a, 2018b). The bioactive compounds of the snake fruit Kombucha (Table 1) can also act as insulin promoters and secretagoues. Insulin promoters are involved in the development of pancreas that aids maturation of $\beta$-cells, whereas insulin secretagoues stimulate insulin secretions from pancreatic $\beta$ cells. Phenolics and tannins have also been shown in in vitro studies to increase insulin secretions from pancreatic $\beta$-cells, and this synergizes with antioxidant compounds in increasing insulin secretions. The rat groups administered with the snake fruit Kombucha and metformin showed the best results and that the snake fruit Kombucha can be an effective diabetes therapy agent.

\section{Conclusions}

The three treatments, the snake fruit Kombucha, black tea Kombucha and metformin, were effective as diabetes therapy agents in the STZ-induced rat model by lowering FPG, improving oxidation stress statuses and lipid profiles. Improvements of pancreas were evident in the higher Langerhans islands and the $\beta$-cells numbers by these three treatments. However, the snake fruit Kombucha was as effective as the metformin in managing the induced diabetes, and more than the black tea Kombucha. Hence, snake fruit Kombucha can potentially substitute metformin as a diabetes therapy agent, and this paves way for an extensive human trial to ascertain this. 


\section{Acknowledgements}

This work was financially supported by the Ministry of Research, Technology and Higher Education, Republic of Indonesia under Grant number 063/SP2H/LT/DRPM/IV/2017.

\section{Conflict of interest}

The authors declare no conflict of interest.

\section{Ethical approval}

The animal study was approved by the Brawijaya University Research Ethics Committee (Ethical Clearance No. KEP-749-UB).

\section{References}

Aloulou, A., Hamden, K., Elloumi, D., Ali, M.B., Hargafi, K., Jaouadi, B., Ayadi, F., Elfeki, A., Ammar, E., 2012. Hypoglycemic and antilipidemic properties of Kombucha tea in alloxan-induced diabetic rats. BMC Complement. Altern. Med. 12, 63-71.

Aralas, S., Mohamed, M., Abu Bakar, M.F., 2009. Antioxidant properties of selected salak (Salacca zalacca) varieties in Sabah, Malaysia. Nutr. Food Sci. 39 (3), 243-250.

Ardheniati, M., Andriani, M.A.M., Amanto, B.S., 2009. Kinetics fermentation on tea kombucha with various types of tea based on its processing. Biofarmasi 7 (1), 48-55.

Beesley, J.E., 1995. Immuno-Cytochemistry: A Practical Approach. Oxford University Press, New York.

Bhattacharya, S., Gachhui, R., Sil, P.C., 2013. Effect of kombucha, a fermented black tea in attenuating oxidative stress mediated tissue damage in alloxan-induced diabetic rats. Food Chem. Toxicol. 60, 328-340.

Dufresne, C., Farnworth, E., 2000. Tea, Kombucha, and health. Food Res. Int. 33, 409-421.

Gordon, M.H., 1990. The mechanism of antioxidant action in vitro. In: Hudson, B.J.F. (Ed.), Food Antioxidants. Elsevier Applied Science, London-New York.

Goh, W.N., Rosma, A., Kaur, B., Fazilah, A., Karim, A.A., Bhat, R., 2012. Fermentation of black tea broth (kombucha): I. Effects of sucrose concentration and fermentation time on the yield of microbial cellulose. Int. Food Res. J. 19 (1), 109-117.

Gunton, J.E., Delhanty, P.C.D., Takahashi, S.I., Baxter, R.C., 2003. Metformin rapidly increases insulin receptor activation in human liver and signals preferentially through insulin-receptor substrat-2. J. Clin. Endocrinol. Metab. 88, 1323-1332.

Iida, K.T., Kawakami, Y., Suzuki, M., Shimani, H., Toyoshima, H., Sone, H., 2003. Effect of thiazolidinediones and metformin on LDL oxidation and aortic endothelium relaxation in diabetic GK Rats. Am. J. Physiol. Endocrinol. Metab. 284, E1125-E1130.

Jasman, I.D., Widianto, D., 2012. Selection of yeast strains for ethanol fermentation of glucose-fructose-sucrose mixture. J. Biotechnol. 17 (2), 114-120.
Jayabalan, R., Malba`sa, R.V., Lon`car, E.S., Vitas, J.S., Sathishkumar, M., 2014. A review on kombucha tea-microbiology, composition, fermentation, beneficial effects, toxicity, and tea tungus. Compr. Rev. Food Sci. Food Saf. 13, 538-550.

Johnson, M.H., De Mejia, E.G., 2016. Phenolic compounds from fermented berry beverages modulated gene and protein expression to increase insulin secretion from pancreatic $\beta$-cells in vitro. J. Agric. Food Chem. 64 (12), 2569-2581.

Kallel, L., Desseaux, V., Hamdi, M., Stocker, P., Ajandouz, E.H., 2012. Insights into the fermentation biochemistry of Kombucha teas and potential impacts of Kombucha drinking on starch digestion. Food Res. Int. 49, 226-232.

Kwon, O., Eck, P., Chen, S., Corpe, C.P., Lee, J., Kruhlak, M., Levine, M., 2007. Inhibition of the intestinal glucose transporter GLUT2 by Flavonoids. FASEB J. 21, 366-377.

Malbasa, R.V., Loncar, E.S., Vitas, J.S., Canadanovic-Brunet, J.M., 2011. Influence of starter cultures on the antioxidant activity of kombucha beverage. Food Chem. 127, 1727-1731.

Ogurtsova, K., Fernandes, J.D.R., Huang, Y., Linnenkamp, U., Guariguata, L., Cho, N.H., Cavan, D., Shaw, J.E., Makaroff, L.E., 2017. IDF diabetes atlas: global estimates for the prevalence of diabetes for 2015 and 2040. Diabetes Res. Clin. Pract. 128, 40-50.

Ostman, E., Granfeldt, Y., Persson, L., Bjorck, I., 2005. Vinegar supplementation lowers glucose and insulin responses and increases satiety after a bread meal in healthy subjects. Eur. J. Clin. Nutr. 59, 983-988.

Rahimi-Madiseh, M., Heidarian, E., Kheiri, S., Rafieian-Kopaei, M., 2017. Effect of hydroalcoholic Allium ampeloprasum extract on oxidative stress, diabetes mellitus and dyslipidemia in alloxan-induced diabetic rats. Biomed. Pharmacother. 86, 363-367.

Scalbert, A., Johnson, I.T., Saltmarsh, M., 2005. Polyphenols: antioxidants and beyond. Am. J. Clin. Nutr. 81 (1 Suppl), 215S-217S.

Srihari, T., Karthikesan, K., Ashokkumar, N., 2013. Antihyperglycaemic efficacy of kombucha in streptozotocin-induced rats. J. Funct. Foods 5 (4), 1794-1802.

Suarsana, I.N., Priosoeryanto, B.P., Bintang, M., 2010. Profile of blood glucose and ultrastucture of betacells pancreatic islet in alloxan compound induced rats. IJAVS 15 (2), 118-123.

Suica-Bunghez, I.R., Teodorescu, S., Dulama, I.D., Voinea, O.C., Simionescu, S., Ion, R.M., 2016. Antioxidant activity and phytochemical compounds of snake fruit (Salacca zalacca). IOP Conf. Ser.: Mater. Sci. Eng. 133 https://doi.org/10.1088/1757-899X/ 133/1/012051. 012051.

Taku, K., Umegaki, K., Sato, Y., Taki, Y., Endoh, K., Watanabe, S., 2007. Soy isoflavones lower serum total and LDL cholesterol in humans: a meta analysis of 11 randomized controlled trials. Am. J. Clin. Nutr. 85, 1148-1156.

Zern, T.L., Fernandez, M.L., 2005. Cardioprotective effects of dietarypolyphenols. J. Nutr. 135, 2291-2294.

Zhang, H., Tsao, R., 2016. Dietary polyphenols, oxidative stress and antioxidant and antiinflammatory effects. Curr. Opin. Food Sci. 8, 33-42.

Zubaidah, E., Dewantari, F.J., Novitasari, F.R., Srianta, I., Blanc, P.J., 2018a. Potential of snake fruit (Salacca zalacca (Gaerth.) Voss) for the development of a beverage through fermentation with the Kombucha consortium. Biocatal. Agric. Biotechnol. 13, 198-203.

Zubaidah, E., Apriyadi, T.E., Kalsum, U., Widyastuti, E., Estiasih, T., Srianta, I., Blanc, P.J., 2018b. In vivo evaluation of snake fruit Kombucha as hyperglycemia therapeutic agent. Int. Food Res. J. 25 (1), 453-457. 